\title{
A Myth Debunked: the Results of SYNTHESIS Expansion and IMS III
}

In February 2013, the results of two key clinical trials assessing the clinical efficacy of endovascular management in acute ischemic stroke were presented at the annual meeting of the International Stroke Conference (ISC) in Honolulu.

The trials, SYNTHESIS expansion and IMS-III, had received great attention from many neurologists and neurointerventionalists, as they were multicenter, randomized, controlled trials comparing clinical results of endovascular approach versus standard intravenous (IV) recombinant tissue plasminogen activator (tPA) alone. Although it had already been demonstrated that endovascular treatment had higher recanalization rates than standard medical care (IV tPA), clinical outcomes of the two methods had not yet been compared directly.

The SYNTHESIS expansion trial, led by Alfonso Ciccone (Carlo Poma Hospital, Mantua, Italy), randomly assigned 362 acute ischemic stroke patients within 4.5 hours of symptom onset to receive IV tPA or intraarterial endovascular treatment, including intraarterial thrombolysis with tPA, mechanical clot disruption or retrieval, or a combination of these interventions. This study was initiated under the hypothesis that "the disadvantage of endovascular treatment in terms of time spent may be offset by more effective recanalization achieved with endovascular therapy." This hypothesis was supported by the preliminary results of the SYNTHESIS study.

The primary outcome of survival (free from disability, modified Rankin score $(\mathrm{mRS}) \leq 1$ ) was assessed at 3 months. $30.4 \%$ of 181 patients given endovascular therapy and $34.8 \%$ of 181 patients given tPA were free of disability ( $\mathrm{mRS} \leq 1)$. This result demonstrated that intraarterial intervention was not superior to standard medical care for patients with acute ischemic stroke. In addition, there were no between-group differences in mortality at 90 days (14.4\% in the endovascular group versus $9.9 \%$ in the IV tPA group, $\mathrm{p}=0.22$ ) or sympto- matic intracranial hemorrhage within 7 days $(6 \%$ in each group).

Notably, patients in the endovascular group did not undergo treatment initiation until one hour after the tPA group, with median onset-to-treatment times of 3.75 hours and 2.75 hours, respectively. In conclusion, the clinical benefit of the endovascular treatment of acute stroke, when compared with IV tPA, could not be proven by the SYNTHESIS expansion trial. However, Ciccone and colleagues presented that generalizability of the results may be limited because demonstrating the presence of an occlusion was not necessary for inclusion in the trial. They said that the selection of patients on basis of the demonstration of vascular occlusion with noninvasive method may provide the endovascular treatment superiority.

Another recent trial, IMS III, is a randomized, multicenter, open-label clinical trial that examined whether combined intravenous and endovascular treatment is superior to standard IV tPA treatment alone for patients with acute stroke. Subjects were randomly assigned to combined or standard therapy within 3 hours of stroke onset in a 2:1 ratio. Endovascular treatment included a choice of catheters and devices or intraarterial tPA based upon lesion characteristics, experience and training of the investigator, and specified use of devices. At the outset, only one device was available, the Concentric Merci retriever, but trial leadership recognized that endovascular technology would evolve. In order to keep the trial clinically relevant and optimize endovascular approach, additional devices including the stent retriever, Penumbra system, and microsonic SV infusion system were allowed as each became cleared for clinical use by the Food and Drug Administration. Contrary to the SYNTHESIS trial, computed tomography angiography (CTA) was routinely used in the identification of occlusion except at the beginning of 


\section{Chang-Woo Ryu}

the trial. According to journal articles, the IMS III study was stopped after 656 participants had been randomized, because it was clear there was no significant difference between the two approaches.

In overall analysis, the 90 day rate of survival free from disability $(\mathrm{mRS}<2$ ) was $30.4 \%$ in the endovascular group and $34.8 \%$ in the IV tPA group, an insignificant difference (OR $0.71 ; 95 \%$ confidence CI, 0.44 to 1.14). There was no statistically significant difference between the interventions for the primary endpoint. The absolute difference between groups was $1.5 \%$ (95\% CI, $-6.1 \%$ to $9.1 \%$ ) when adjusted for stroke severity by the National Institutes of Health Stroke Scale (NIHSS) score. Although high recanalization rates using the endovascular approach were achieved with active use of a thrombectomy device in this study, the clinical outcome was disappointing. This result of IMS III also demonstrated that the effective recanalization rate of endovascular therapy cannot offset the time required for completion of endovascular treatment. Additionally, it was concluded that bridging therapy should not be considered routine protocol but instead should be thought of as a highly-selective therapeutic option.

Despite the fact that these two trials comprise research on the extended application of endovascular approach in overcoming standard medical treatment, the management guidelines for acute ischemic stroke have remained unchanged. However, the merit of the bridging method, which has been commonly used to recanalize large artery occlusions, was brought into question by these trials. The window of time during which endovascular approach may be attempted (4.5$6 \mathrm{~h}$ ) is so narrow that the amount of patients for which this procedure may be indicated was significantly decreased. With fewer patients receiving endovascular recanalization therapy, there is less opportunity for trainees to gain experience in intraarterial thrombectomy, and hospitals or medical insurance companies may hesitate to use willingly the money for maintenance of the emergent neuroendovascular system. Even more damaging, the ability to recruit patients to new trials that assess the superiority of endovascular treatment will be difficult.
Not every result was unfriendly to endovascular treatment, however. In IMS III, for predefined subgroups of patients with an NIHSS score of 20 or higher, indicating severe stroke (endovascular therapy, $23.5 \%$; IV tPA alone, $16.7 \%, \mathrm{p}=0.07$ ), comparison of the functional outcomes between the endovascular and medical groups showed a tendency toward a better outcome in the endovascular group. This result may indicate that more severe stroke associated with proximal arterial occlusion, including carotid $\mathrm{T}$ and proximal M1, would potentially have a greater benefit with the endovascular approach, as these clot locations may be highly amenable to endovascular therapy. The role of endovascular therapy is considered to be more critical in proximal artery occlusion than distal artery occlusion. To this end, it would be helpful if IMS III investigators additionally report result of subgroup comparisons using CTA. Each time encountering the patients with acute stroke, we will be conflicted between our empirical knowledge and verified evidence.

The myth has been debunked, but the challenge is still present. The endovascular therapy system that is able to start endovascular approach as soon as the patient come to hospital and more effective thrombectomy device such as stentriever, will allow the endovascular treatment to play critical role in treatment of stroke.

\section{References}

1. Broderick JP, Palesch YY, Demchuk AM, Yeatts SD, Khatri P, Hill MD, et al. Endovascular therapy after intravenous t-pa versus t-pa alone for stroke. $N$ Engl J Med 2013 Feb 7. [Epub ahead of print]

2. Ciccone A, Valvassori L, Nichelatti M, Sgoifo A, Ponzio M, Sterzi R, et al. Endovascular treatment for acute ischemic stroke. N Engl J Med 2013 Feb 6. [Epub ahead of print]

3. Ciccone A, Valvassori L, Ponzio M, Ballabio E, Gasparotti R, Sessa M, et al. Intra-arterial or intravenous thrombolysis for acute ischemic stroke? The SYNTHESIS pilot trial. J Neurointerv Surg 2010;2:74-79

\section{Chang-Woo Ryu}

Editor-in-Chief, Neurointervention Department of Radiology, Kyung Hee University Hospital at Gangdong, Seoul, Korea 